\title{
Variación de estilo en interacciones digitales: huéspedes y hoteles en reseñas TripAdvisor
}

\section{Style variation in digital interactions: guests and hotels in TripAdvisor reviews}

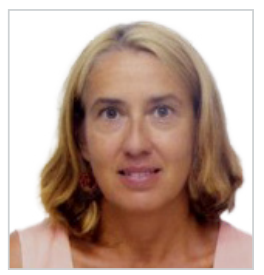

Raquel Hidalgo Downing. Doctora en Filología Hispánica, ha sido Premio Extraordinario de Doctorado UCM. Es Profesora Titular del área de Lingüística en la Universidad Complutense de Madrid. Dirige el Grupo de Investigación UCM Lingüística, Discurso, Corpus: aplicaciones tecnológicas y didácticas. Es miembro del Proyecto Variación pragmática en la expresión de la cortesía en español y es IP (con Prof. Iglesias Recuero) del Proyecto I+D+ i Los procesos de la gestión de la imagen y la (des)cortesía: perspectivas históricas, lingüísticas y discursivas. Dirige (con Prof. Joaquín Garrido Medina) la colección Lingüística y Comunicación de la Editorial Complutense. Sus principales líneas de investigación son la pragmática y el análisis del discurso, y la lingüística aplicada a la enseñanza. Ha publicado recientemente La competencia pragmática en ELE (M. Regueiro, Semántica y pragmática en ELE. Edinumen, 2020), Márquez-Reiter \& Hidalgo Downing, Intercultural Communication in a globalized world (en D. Koike \& C. Félix-Brasdefer, Handbook of Spanish Pragmatics, 2020). Universidad Complutense de Madrid, España rhidalgo@ucm.es ORCID: 0000-0003-4930-7903

Recibido: 29/06/2020 - Aceptado: 14/10/2020

\section{Resumen:}

En este trabajo se propone un análisis del estilo comunicativo que aparece en reseñas realizadas por huéspedes de hoteles y sus consiguientes respuestas, en la popular plataforma de viajes y turismo TripAdvisor. El objetivo es identificar y analizar el conjunto de rasgos lingüísticos de estilo que aparecen tanto en las opiniones de los usuarios como en las respuestas de los responsables de las empresas hoteleras. Si bien en los inicios de los estudios sobre comunicación mediada por ordenador se identificó un conjunto de rasgos generales que podían asociarse típicamente a este tipo de comunicación, especialmente en torno a la oralidad e informalidad, estudios posteriores han mostrado la enorme variación que aparece en este tipo de comunicación, por lo que resulta imposible ofrecer una visión general del español en internet, que en cambio ha de situarse en la observación de prácticas discursivas concretas. A partir del análisis de la variación de estilo sobre un corpus de reseñas y respuestas, el estudio muestra que tanto huéspedes como hoteles exhiben rasgos de adaptabilidad al medio para alcanzar
Received: 29/06/2020 - Accepted: 14/10/2020

\section{Abstract:}

In this article we analyse variation in communicative style that appears in guests' reviews and hotel responses on TripAdvisor, the popular travel and tourism platform. The aim of the study is to examine style features that appear in guests' opinions and hotel responses. Although many studies in computer-mediated communication have identified informality and orality as key features in this type of communication, more recent studies claim the important communicative and linguistic variation that appears in language in CMO, making it impossible to offer an overview of Spanish on the internet, which instead must be observed in situated discourse practices. The analysis of style variation on a corpus composed of reviews and their responses shows that both guests and hotels exhibit traits of adaptability to the medium in order to achieve their communicative goals, but show different and divergent, if not opposed, communication styles, which reflect the users' social realities and images, and

\footnotetext{
Cómo citar este artículo:

Hidalgo Downing, R. (2020). Variación de estilo en interacciones digitales: huéspedes y hoteles en reseñas TripAdvisor. Doxa Comunicación, 31, pp. 361-380.
} https://doi.org/10.31921/doxacom.n31a18 
sus finalidades comunicativas, pero muestran estilos comunicativos diferentes y divergentes, por no decir encontrados y opuestos, reflejo de las realidades e imágenes sociales de los usuarios y de las audiencias potenciales que posee cada uno, enfrentando percepciones polivalentes de la norma lingüística al tiempo que ampliando las posibilidades de innovación y expresión lingüística.

Palabras clave:

Variación de estilo, reseñas de consumo, comunicación digital, pragmática en internet, norma lingüística. the potential audiences to whom interactions are addressed, ultimately projecting different perceptions of linguistic standards, while expanding the possibilities of linguistic innovation and expression.

Keywords:

Style variation; consumer reviews; digital communication; internet pragmatics; language standard.

\section{Introducción ${ }^{1}$}

En el ámbito de la comunicación mediada por ordenador ha aumentado el interés en los últimos años por estudiar las interacciones que se desarrollan en distintos ámbitos, como el consumo, el turismo y el viaje, gracias al denominado boca a boca electrónico. Desde la plataforma 2.0 la difusión de la opinión del usuario se despliega con una enorme variedad, ya sea en forma de opinión o comentario tras la lectura de una noticia, una columna periodística, una nota editorial, como tras la adquisición de productos de consumo (Chen \& Xie, 2008). En este contexto de interacción abierta y horizontal emerge la figura del "prosumidor", el consumidor que además es productor de contenidos en la red (Vasquez, 2011, 2014; Virtannen, 2017).

En este trabajo nos detenemos en este fenómeno, en concreto en la reseña de huéspedes que comentan su estancia en hoteles a través de una plataforma como TripAdvisor, creada precisamente para albergar las opiniones de los usuarios. La plataforma ha permitido que las experiencias de los huéspedes se compartan con otros viajeros, a la vez que son recibidos y leídos por los responsables de los hoteles (Law 2006). Esta dualidad de destinatarios convierte las opiniones de TripAdvisor en una forma de publicidad gratuita para las empresas, que ven así difundidos sus servicios por iniciativa propia de los usuarios (Miguéns, Baggio \& Costa, 2008).

El estudio de las opiniones de usuarios ha despertado un interés creciente en los estudios de pragmática y discurso; en particular, se han descrito las formas de queja que se desarrollan en las plataformas TripAdvisor (Vasquez, 2011, 2014) y de satisfacción del cliente en Airbnb (Hernández López, 2019), si bien siempre sobre el inglés. En español, ha despertado el interés estudiar los mecanismos de atenuación que mitigan los actos de habla que aparecen en las reseñas hoteles y restaurantes (Mancera Rueda, 2018, Hernández Toribio y Mariottini, 2015), así como los procesos de narración de experiencias por parte de los viajeros (Mariottini y Hernández Toribio, 2017).

Sin embargo, las respuestas a las opiniones de usuarios apenas han recibido atención, cuando estas constituyen formas reactivas que se emparentan directamente con la reseña y que permiten ver la práctica discursiva en su totalidad. Además, mientras que la mayoría de los estudios se han centrado en actos de habla y en particular en las quejas, o bien en aspectos concretos como la atenuación, no se ha analizado el modo en que transcurren las interacciones entre huéspedes

1 El estudio forma parte de la investigación realizada en el marco del proyecto de investigación titulado "Variación pragmática en la expresión de la cortesía en español” financiado por UCM-Grupo Santander, REF PR87/19-2254, fecha de concesión 4/12/2019, así como del I+D+ i titulado Los procesos de la gestión de la imagen y la (des)cortesía: perspectivas históricas, lingüísticas y discursivas, PID2019-107668GB-I00, fecha de concesión 20/06/2020. 
y hoteles, como tampoco sus rasgos de estilos y de registros. Otra cuestión interesante que surge en la recogida de reseñas, como en toda recopilación de datos de internet, es en qué medida ha de delimitarse la práctica discursiva para poder acotar la metodología de estudio. Por todo ello, el objetivo de este estudio es observar las interacciones que se desarrollan entre opiniones de usuarios y respuestas y estudiar los rasgos estilísticos de unos y otros. La hipótesis del trabajo es que no existe homogeneidad en el estilo comunicativo de los usuarios de la plataforma, sino variación e incluso abierta divergencia, en consonancia con las finalidades comunicativas de los usuarios y de la relación que establecen con prácticas discursivas fuera de la red, offline. Una cuestión importante del corpus presentado es la relación de los rasgos estilísticos de huéspedes y hoteles con las nociones y percepciones de la norma lingüística, lo que puede llevar a la discusión sobre cómo considerar este tipo de escritura digital en relación con el concepto de norma.

El artículo se organiza de la manera siguiente: en primer lugar, se revisa la bibliografía sobre el tema. En la sección 3, se discuten algunos aspectos relativos a la noción de estilo y registros, y los parámetros con los que puede estudiarse. En la sección 4, se introducen los datos y la metodología. En las secciones 5 y 6, se presentan los resultados y discusión del análisis de los distintos parámetros estilísticos escogidos que emplean los huéspedes autores de las reseñas y las respuestas de los hoteles desde un enfoque comparado.

\section{Las reseñas de opinión en internet}

Las reseñas de consumo en internet constituyen un género emergente que surgió a través de breves comentarios que ofrecían los usuarios tras la adquisición de productos por internet (a través de los servicios de Amazon) y que se han extendido a numerosas áreas del comercio, creando un género discursivo en el que el consumidor es quien transmite información y opinión sobre el producto (Chen \& Xie, 2008). Vasquez $(2011,2014)$ describe a los autores de reseñas como "prosumidores", un término que alude a los consumidores que son a la vez productores, normalmente no remunerados, de contenido online.

Las reseñas de consumo han recibido atención en distintas disciplinas, principalmente el marketing y la publicidad, campos en los que el estudio de las opiniones de los usuarios está muy extendida, dada la utilidad de sus resultados para el diseño de las estrategias publicitarias de las empresas (Brigs, Sutherland \& Sioban, 2007; Sparks \& Browning, 2010). En estos estudios, se ha mostrado que los usuarios leen con atención las opiniones y valoran más los productos que incluyen las opiniones de otros usuarios (Munar \& Jens, 2014; Miguéns, Baggio \& Costa, 2008). En los estudios de orientación lingüística, se ha prestado atención preferente al análisis de las reseñas negativas y las quejas, en inglés fundamentalmente (Vasquez, 2011, 2014, Zhang \& Vasquez, 2014). En español, Mancera Rueda (2018) ha estudiado los mecanismos de atenuación en TripAdvisor a partir de un corpus de reseñas de hoteles y restaurantes de distintos lugares, mientras que Hernández Toribio y Mariottini (2015) describen los actos de habla que aparecen en las opiniones de los usuarios, y profundizan además en la construcción de la narración de experiencias de viajeros en TripAdvisor (Mariottini y Hernández Toribio, 2017). Además, se ha tratado de forma conjunta el análisis de opiniones negativas y positivas con un enfoque pragmático comparado y la cuestión de la interculturalidad en las experiencias de viajeros en el mundo hispánico (Márquez-Reiter e Hidalgo Downing, 2020). 
Para describir algunas características generales de las reseñas de consumo, Virtanen (2017: 81) se sirve de la noción de adaptabilidad enunciada por Verschueren:

"The property of language which enables human beings to make negotiable linguistic choices from a variable range of possibilities in such a way as to approach points of satisfaction for communicative needs" (Verschueren, 1999: 61)

En la comunicación mediada por ordenador (CMO) o, mejor, comunicación mediada por tecnología o CMT (Gianmateo, Gubitosi y Parini, 2017)2 ${ }^{2}$ la adaptabilidad se traduce en un conjunto de posibilidades y limitaciones que ofrece el entorno (lo que en inglés denominan "affordances"), como son el locus (contexto), el dinamismo (los procesos) o las audiencias. En cuanto al primero, las reseñas de consumo poseen una clara finalidad comunicativa, que es la de evaluar un producto de consumo y por ello adoptan una orientación hacia el tópico o hacia el autor; es decir, se construyen en torno al tema objeto de opinión (tópico-opinión) o bien en torno a la percepción del autor (yo-opinión).

Orientada al tópico

(1) El libro perfecto para enseñar sintaxis, salvo por un detalle. No incluye las soluciones a los ejercicios, lo que habría sido muy útil.

Orientada al autor

(2) En mi opinión, el libro XX es una introducción excelente al estudio de la pragmática. Una lástima que el curso que estoy enseñando termine en enero.

(Adaptado de Virtanen, 2017: 82)

La autora analiza un corpus de reseñas sobre manuales de lingüística, por lo que esas características (orientación al tópico/orientación al autor) son particularmente marcadas, y la adaptabilidad se muestra aquí como un nexo entre los géneros académicos (la audiencia a la que se dirige) y los promocionales, ya que las reseñas están escritas por expertos en la materia, que muestran sus conocimientos, pero tienen también una vocación de difusión persuasiva. En otros tipos de reseñas, como las de hoteles que ocupan este trabajo, el autor de la reseña evalúa una experiencia, no un producto, que por tanto ha de ser vivida antes de poder ser evaluada (Vasquez, 2011, Mariottini y Hernández Toribio, 2017) y puede adoptar formas distintas, ya sea como opinión breve, narración, argumentación. El estudio de las respuestas, que no está incluido en el trabajo de Virtaanen ni en el de Vasquez, permite indagar aún más en las posibilidades del 'locus'; las formas reactivas a las opiniones de los prosumidores aportan el punto de vista de las empresas, cuyas finalidades comunicativas y diseño específico de audiencias se proyectan tanto hacia la comunidad interna (online) como externa (offline) a la red. En nuestro corpus, por tanto, el objetivo principal es precisamente estudiar las reseñas y sus respuestas de forma conjunta, para poder describir los procesos y representaciones que tienen lugar en estas interacciones.

2 La evolución de los soportes informáticos y la irrupción de los dispositivos móviles en la vida diaria y la comunicación conlleva un conjunto de cambios importantes, que afectan también al ámbito de la comunicación. Según Gianmateo, Gubitosi y Parini (2017), hoy día resulta más adecuado hablar de comunicación mediada por tecnología, un término que abarca el uso de cualquier dispositivo electrónico para la comunicación. 


\section{La noción de estilo en la comunicación mediada por ordenador}

Tal y como la investigación sociolingüística y sociopragmática de las últimas décadas ha mostrado, la elección de un estilo en una actividad comunicativa depende de la relación -socialmente establecida- entre las características lingüísticas formales del enunciado y la propiedades de la situación comunicativa; por ello, tiene consecuencias para la representación que hablantes y destinatarios se forman de la experiencia en que es adecuado dicho uso. La práctica social, ya sea pública o privada, las condiciones de producción y recepción, el canal, la naturaleza interpersonal o institucional de la actividad comunicativa, el esquema de participación, las relaciones de jerarquía y solidaridad existentes entre los participantes, -es decir, lo que Halliday (1985) subsumía en los conceptos de campo, tenory modo- determinan las características estilísticas de las distintas modalidades discursivas. De acuerdo con esta idea (Halliday, 1985; Gee, 1999; Garrido Medina, 1997: 114117), el estilo no designa simplemente un conjunto de propiedades lingüísticas que se superpone a un contenido previo; el estilo es significado -lo transmite- en la medida en que su uso refleja la representación que los hablantes tienen del contexto de situación, y por tanto, de cómo deben interpretarse los enunciados en él. Por tanto, la elección de un estilo está asociada a contextos de uso particulares y a las formas en que los hablantes de una comunidad lingüística se representan -a partir de expectativas socialmente construidas- el funcionamiento de los componentes de dichos contextos. Los registros, en cuanto variedades lingüísticas asociadas a propiedades más generales de las interacciones, desempeñan un papel fundamental en la configuración del estilo. Mientras que algunos autores hacen coincidir los rasgos de etilo con sociolecto o rasgos de la variación social, otros destacan que un mismo grupo social puede presentar distintos estilos, por lo que cabe considerar el estilo como una categoría contextual, que pone en relación los rasgos situacionales con los lingüísticos (Garrido Medina, 1997: 114). El estilo se caracteriza por la posibilidad de elección, mientras que la variación social puede estar determinada al menos parcialmente por el usuario, y no (no solo) por el uso. En la variación de uso, el estilo se refiere "al conjunto de factores que intervienen en la situación comunicativa, y se coincide en considerar fundamental la relación con el oyente, es decir el diseño del destinatario o público receptor, la acomodación al otro, la interacción en redes sociales, o el prestigio en el 'mercado lingüístico'” (Garrido Medina, 1997: 117). Esta concepción del estilo es particularmente relevante para nuestro corpus porque, como se verá en el análisis, los estilos comunicativos reflejan representaciones distintas acerca de las expectativas de lo que ofrece el medio y de las intenciones que poseen los usuarios de la plataforma en cuanto al diseño de las audiencias.

En la comunicación mediada por tecnología, la ya sólida tradición de estudios ha destacado las características estructurales de la comunicación por internet (véase Herring et. al., 2013; Yus, 2011), tales como la interactividad, la horizontalidad o la multimodalidad (Herring et. al., 2013). Algunos de los rasgos que se han asociado a este tipo de comunicación son la informalidad y oralidad (Yus, 2011; Vela Delfa, 2016) y el alejamiento de la norma lingüística (Mancera Rueda, 2016), en consonancia con la irrupción de una forma de escritura mínima e inmediata, sin filtros normativos. Sin embargo, en las últimas décadas los estudiosos observan cómo las prácticas discursivas se modifican continuamente, en consonancia con la rápida evolución de la red. Un caso paradigmático es el correo electrónico; inicialmente se describió como un género informal, con rasgos que se asociaban a la oralidad (Yus, 2011), mientras que estudios más recientes lo describen en entornos profesionales y académicos (Pérez Sabater, Turney y Montero, 2008). La claridad, economía y expresividad se destacan como elementos característicos del estilo comunicativo de mensajes de texto SMS (Cantamutto, 2017); estos 
tres rasgos responden de forma evidente a los factores contextuales que configuran este tipo de texto (brevedad y rapidez de respuesta o reacción), y explican también la abundancia de formas abreviadas y emoticonos. Cantamutto (2017) menciona, además, la informalidad, rasgo que se destaca de forma general para la comunicación mediada por ordenador (Herring et. al, 2013; Yus, 2011). No obstante, otros trabajos han matizado la identificación de rasgos que pueden aplicarse sin tener en cuenta un análisis pormenorizado de los participantes en las interacciones. A partir de un corpus de correos electrónicos en inglés que se desarrollan en el ámbito académico, en concreto entre profesores de universidad, Pérez Sabater, Turney y Montero (2008) estudian la aparición y frecuencia de parámetros de estilo como los saludos, las fórmulas de cortesía, el uso de emoticonos y emojis, las formas abreviadas y contracciones. Los autores encuentran variación importante entre los correos electrónicos individuales (dirigidos a un solo destinatario) y los colectivos (dirigidos a varios destinatarios). Mientras que los primeros exhibían rasgos de informalidad y apenas contenían marcadores de cortesía o saludos elaborados, los colectivos en cambio recordaban al formato de carta profesional, con saludos formales y fórmulas de cortesía. En ninguno de los dos tipos encontraron uso significativo de formas abreviadas o emoticonos. Estas diferencias estilísticas sugieren que, pese a la presencia de determinados rasgos propios de la comunicación mediada por tecnología, los correos electrónicos, como otros ejemplos de interacciones digitales, "reflejan las realidades sociales de sus usuarios" (Herring et. al. 2013: 11). Por tanto, estos estudios muestran que en realidad se producen diferencias muy notables entre prácticas interactivas en la red. Tal y como defienden Ngwenyama \& Lee (1997), las interacciones digitales no transmiten simplemente determinados rasgos lingüísticos, estructurales o textuales, sino que reflejan, a la vez que producen, prácticas discursivas dinámicas en las que se ponen en marcha y que crean relaciones sociales:

When people communicate, they do not send messages as electronically linked senders and receivers. They perform social acts in action situations that are normatively regulated by, and already have meaning within, the organizational context. As organizational actors, they simultaneously enact existing and new relationships with one another as they communicate. (Ngwenyama \& Lee, 1997: 164).

En nuestro corpus, las interacciones entre huéspedes y hoteles muestran que los huéspedes actúan socialmente al compartir con una audiencia el relato de su estancia, y los hoteles no "responden" en el sentido estrictamente textual a las opiniones de los usuarios, sino que actúan pragmática y discursivamente para establecer (nuevas) relaciones con los usuarios/huéspedes, que remitan y tengan repercusión sobre prácticas offline. La comunicación a través de la plataforma permite la interacción con distintos "papeles de recepción" (Goffman 1981): los destinatarios directos (los viajeros que redactaron la reseña) y los indirectos (aquellos que también redactaron reseñas pero no esa concreta a la que contesta el hotel) Portolés (2004: 223-226), siguiendo a Goffman, distingue entre estos oyentes directos e indirectos, y además habla de oyentes casuales o furtivos, a los que no se dirige el mensaje pero que pueden escucharlo o leerlo, fenómeno que también recoge Albaladejo (2010: 928) en su estudio sobre la poliacroasis, que define como "la recepción e interpretación plural de los discursos retóricos". En este caso, TrypAdvisor permite la presencia de oyentes casuales o furtivos, que leen habitual o esporádicamente las reseñas; sin duda, los hoteles tienen en cuenta también la presencia de esos usuarios, potenciales clientes. No obstante, Albadalejo (2010: 930-931) describe el fenómeno refiriéndose a las menciones a esos usuarios casuales en los discursos (de Barack Obama), mientras que en nuestro corpus no hemos encontrado en las respuestas de los hoteles alusión a esos otros usuarios. 
En cuanto a la norma, Mancera Rueda (2016) estudia los usos que se alejan de la norma y distingue dos tipos, los que vulneran las normas ortográficas (errores ortográficos) y los que se emplean de forma consciente con una intención comunicativa y expresiva (heterografía u ortografía alternativa). Este segundo tipo responde al propósito de introducir rasgos innovadores en la escritura digital, mediante la imitación o adaptación de la expresividad oral; por ejemplo, el uso de varios signos de exclamación o interrogación (Mancera Rueda, 2016: 10). La autora considera que algunos usos están consolidados en la comunicación digital, como el uso de abreviaturas ( $x q$ en lugar de porque), y no constituyen errores sino formas intencionadas y extendidas en la comunicación digital. Al hilo de estas consideraciones, y de acuerdo con la tradición coseriana, el concepto de norma posee al menos dos sentidos principales (entre otros, puesto que se trata de un concepto polisémico): (i) el sentido prescriptivo, como aquello que se debe decir o escribir y se asocia con la corrección lingüística, y (ii) la norma en sentido descriptivo, como lo que es usual o habitual (Martin Zorraquino 1988: 431-440). El primero recoge el sentido de corrección y remite a los hábitos lingüísticos respaldados en formas autorizadas y prestigiadas de la lengua y sus usuarios, normalmente en prácticas discursivas públicas. El segundo sentido de norma, en cambio, hace alusión a lo que es normal o usual. Los dos sentidos de norma se relacionan de una manera tal que la segunda precede a la primera, es decir los hábitos lingüísticos se hacen usuales o habituales, y a continuación, se codifican en la lengua; es decir, aparecen en las gramáticas, los manuales de uso y las ortografías (Méndez García de Paredes 1999: 111).

\section{Metodología y datos del estudio}

El estudio de la comunicación mediada por tecnología contempla distintos métodos de investigación y recogida de datos; una orientación sumamente interesante es aquella que emplea datos cuantitativos a gran escala, los denominados big data, que permite la descarga de datos masivos y la observación de fenómenos comunicativos o lingüísticos en plataformas de internet. De Benito y Estrada Arraz (2018) estudian rasgos fónicos y morfosintácticos, que relacionan con la geolocalización, para extraer datos sobre variación. Esta orientación posee un indudable interés, pero también algunas limitaciones, como la heterogeneidad de las interacciones y los usuarios recogidos (que no seleccionados), por lo que resulta sumamente difícil contextualizar los fenómenos estudiados.

Otra aproximación es la compilación de datos en un corpus a escala menor pero que posea una coherencia interna que permita la observación de prácticas discusivas en internet. Este estudio sigue esta orientación y se ha realizado tras la compilación y selección de un corpus de reseña de hoteles de cadenas españolas en los principales destinos turísticos (Ministerio de Comercio y Turismo, Gobierno de España, 2019). En este trabajo, el propósito es centrar el análisis en un corpus de reseñas en español, escritas por autores hispanohablantes nativos, por lo que se ha escogido trabajar sobre los destinos de habla hispana y descartar otros destinos por el momento. ${ }^{3}$ Por otro lado, en lugar de recoger solo evaluaciones negativas, como en el estudio de Vasquez (2011), se ha optado aquí por recoger todas las evaluaciones en un periodo de tiempo determinado (octubre 2018-marzo 2019), obteniendo un corpus de 120 reseñas y 102 respuestas. Siguiendo los estudios realizados sobre rasgos estilísticos, como Sabater, Turney y Montero (2008) o Mancera Rueda (2016), se han

3 Se ha escogido un conjunto de 3 cadenas hoteleras con distinta clasificación, de 5 a 3 estrellas, a las que se referirá, por razones de anonimización, como A $\left(5^{*}\right), \mathrm{B}\left(4^{*}\right)$ y C $\left(3^{*}\right)$. 
seleccionado los siguientes parámetros de estudio: (i) saludos y despedidas, (ii) formas de tratamiento, (iii), fórmulas de cortesía, y (iv) rasgos ortotipográficos. Para ello, se ha etiquetado el corpus completo y se ha realizado un análisis manual y automático mediante el software Sketch Engine, calculando las apariciones de los elementos estudiados y la ratio de apariciones por reseña. La selección de parámetros no pretende tanto la exhaustividad como la posibilidad de observación en las interacciones entre autores de reseñas y hoteles. La hipótesis de trabajo es que el corpus presenta variación de estilo entre las reseñas y las respuestas, y que estas diferencias se relacionan con las intenciones comunicativas de los participantes en la plataforma. En las secciones que siguen, se presentarán los resultados del análisis, así como una discusión sobre las diferencias encontradas y su relación con las nociones de la norma lingüística.

\section{Análisis de los rasgos de estilo en reseñas y respuestas}

En la Tabla 1 se muestran los resultados del cómputo de apariciones y ratio de saludos y despedidas que aparecen en las reseñas y respuestas de los hoteles. Estas constituyen formas de apertura y cierre de las interacciones que aportan información relevante sobre las características socio-pragmáticas de los participantes y la relación que establecen, ya que a través de ellos, marcan que son "actores sociales" que desempeñan un rol en la interacción y que muestran un grado determinado de confianza o distancia con respecto a los interlocutores (Briz, 1998), por lo que aportan indicios acerca del campo y del tenor funcional e interpersonal (Halliday, 1985). En el corpus que nos ocupa, la presencia (o no) de aperturas y cierres es particularmente relevante, puesto que la reseña constituye un género discursivo emergente en el que no existe una configuración completamente establecida. La respuesta, en cambio, constituye una "segunda parte del par" que posee unos rasgos más identificables, puesto que ha de remitirse y relacionarse intertextualmente con la reseña inicial.

Tabla 1. Saludos y despedidas en reseñas y respuestas de hoteles. Número de apariciones y [ratio de apariciones por reseña] respuestas de hoteles

\begin{tabular}{|l|c|c|c|c|}
\hline \multirow{2}{*}{ Formas } & \multicolumn{2}{|c|}{$\begin{array}{c}\text { Reseñas } \\
(120 \text { analizadas })\end{array}$} & \multicolumn{2}{c|}{$\begin{array}{c}\text { Respuestas hoteles } \\
\text { (102 analizadas) }\end{array}$} \\
\hline & $\mathrm{N}^{\mathrm{a}}$ & {[]} & $\mathrm{N}^{\mathrm{a}}$ & {[]} \\
\hline Saludos & 4 & {$[0,02]$} & 102 & {$[1]$} \\
\hline Despedidas & 5 & {$[0,05]$} & 101 & {$[0,99]$} \\
\hline
\end{tabular}

Fuente: Elaboración propia

Los resultados muestran que los autores de las reseñas apenas emplean formas de iniciación o saludos (solo 4 casos de saludos) frente a los hoteles, que presentan formas de saludo y despedida de forma sistemática. Cuando los autores de reseñas emplean el saludo, lo hacen con la opción informal (4) o semi-formal (3), y por tanto se dirigen genéricamente a los usuarios de la plataforma. Los autores de las reseñas no emplean formas de despedida salvo ocasionalmente (5), pero sí cierran la reseña en algunos casos con fórmulas de (no) recomendación, que marca tanto el cierre como el resumen o síntesis de la evaluación (6). 
(3)

Buenas tardes a todos, el pasado 24-25 y 26 de julio fuimos al Hotel C de Mallorca. (Opinión 5 Hotel C)

(4)

Hola! Me alojé en el hotel del 1 al 10 de Febrero del 2018. Tengo opiniones encontradas (Opinión 13 Hotel B)

(5)

Excelentehotelyexcelentelaatencióndesupersonal(todosnostrataronmuybienyseesforzaronparaquenuestraestadíaseaúnica, especialmente Samuely Karina en Coco’s beach club). También La Palapa bar es otro muy buen lugar que nos trataron muy bien. Saludos. (Opinión 5 Hotel A)

(6)

Pero estan en terrible estado todo, las camas, los closet los baños, las camas ni ganas de dormi dan de ver los rodapies tan sucios y las ropas de camas tan viejas que no sabes si estan sucias oh solo viejas!!!!... no lo recomendaria para hospedarse!!!!4 (Opinión 10 Hotel C)

En las respuestas de los hoteles, en cambio, la aparición sistemática de saludos y despedidas conforma un rasgo característico de la respuesta, que la identifica como tal. El saludo siempre es formal y epistolar, comenzando con "estimado/a" en todos los casos, o la variante "apreciado", y sigue la fórmula de saludo genérico, "estimado huésped" o "apreciado huésped", o bien personalizado, "estimado XXX", reproduciendo el nombre que aparece en el perfil o la firma del autor de la reseña. El saludo se apoya además en fórmulas de cortesía como agradecimientos y disculpas, que conforman los primeros enunciados de la respuesta, y dotan a la respuesta de un estilo formal y elaborado, que recuerda inequívocamente el modelo de carta escrita, y específicamente, de carta comercial. En cuanto a las despedidas, que también aparecen de forma sistemática, las respuestas de los hoteles contienen una despedida compleja, que contiene una fórmula de cortesía, normalmente una invitación a regresar al hotel (le invitamos a volver) y el saludo propiamente dicho, siempre formal, como "atentamente", "cordialmente", acompañado de la firma, con el nombre de la persona que ha escrito la respuesta y su cargo en la empresa (Director Comercial, Community Manager, Gerente de Calidad).

(7)

Opinión 5 Hotel B)

Familia XXX

Lugar muy hermoso, bien ubicado, grato ambiente, rica comida e instalaciones apropiadas. Amabilidad de la mayoría de sus trabajadores. De todas formas, para nosotros sigue siendo mejor Barceló Dominican Beach sobre Ociidental Cancun y Occidental Cartagena de Indias.

4 Los textos se reproducen inalterados, sin corregir, para mantener los rasgos originales de los mismos. 
Estimada Familia XXX

Agradecemos haberse tomado el tiempo para dejarnos los comentarios de su última estancia con nosotros y nos haya compartido su grata experiencia, es nuestra prioridad ofrecer experiencias memorables a todos nuestros huéspedes proporcionando el legendario servicio y la calidez que nos caracteriza.

Esperamos muy pronto poder darle la bienvenida nuevamente a este pequeño paraíso en Cancun.

Saludos Cordiales,

XXX.

Asistente de Gerencia

Tal y como puede observarse en (7), mientras que el autor de la reseña no emplea el saludo ni la despedida sino que orienta la reseña al esquema tópico + opinión (Virtanen 2017), el hotel se dirige específicamente al autor (Estimada Familia $X X X)$, mediante un saludo formal y epistolar, y cierra la interacción con una secuencia compuesta por una fórmula de invitación (esperamos muy pronto poder darle la bienvenida nuevamente), el saludo formal (saludos cordiales) y la firma (nombre y cargo de la persona en la empresa). De ello se extrae que el autor de la reseña no emplea formas apelativas y no dirige su opinión de forma específica, salvo a otros usuarios de la plataforma, mientras que los hoteles establecen una relación directa con el cliente (mencionando su nombre) pero con un estilo formal y distanciado que destaca el carácter profesional de la respuesta y la proyección de una imagen corporativa que sea vista por el cliente escogido y por todos los usuarios de la plataforma.

El segundo parámetro analizado es el uso de las formas de tratamiento, formas pronominales y personales del verbo, tanto en las reseñas como en las respuestas de los hoteles, cuyos resultados aparecen en la Tabla 2. Para ello se han computado tanto los pronombres personales (yo, tú, etc.) como las marcas de persona en los verbos.

Tabla 2. Formas de tratamiento en reseñas y respuesta de hoteles. Número de apariciones y [ratio de apariciones por reseña] respuestas de hoteles

\begin{tabular}{|l|c|c|c|c|}
\hline \multirow{2}{*}{ Formas } & \multicolumn{2}{|c|}{ Reseñas } & \multicolumn{2}{c|}{ Respuestas hoteles } \\
\hline & $\mathrm{N}^{\mathrm{a}}$ & {[]} & $\mathrm{N}^{\mathrm{a}}$ & {[]} \\
\hline 1 persona singular (yo) & 340 & {$[2,83]$} & 21 & {$[0,21]$} \\
\hline 2 persona singular (tú) & 70 & {$[0,68]$} & - & - \\
\hline 1 persona plural (nosotros) & 449 & {$[3,74]$} & 589 & {$[5,77]$} \\
\hline 2 persona plural (vosotros) & 36 & {$[0,30]$} & - & - \\
\hline Usted/le & 4 & {$[0,03]$} & 375 & {$[3,68]$} \\
\hline Ustedes/les & 2 & {$[0,02]$} & 15 & {$[0,15]$} \\
\hline Sr./Sra./Sres. & 5 & {$[0,05]$} & 8 & {$[0,08]$} \\
\hline
\end{tabular}

Fuente: Elaboración propia

5 El corpus ha sido sometido a un proceso de anonimización, por lo que los nombres de empresas o personas no aparecen. 
Tal y como puede observarse, los autores construyen la opinión en torno a su experiencia o valoración personal, por lo que una fórmula frecuente es aquella que expresa yo + opinión y que explica por tanto la aparición de la primera persona en el corpus (340 casos de "yo" y 440 de "nosotros"). Esta fórmula de yo+ opinión (7) cambia a la primera persona del plural, nosotros, cuando el autor de la reseña desea reflejar que la estancia fue compartida con familia o amigos (8):

(8)

Yo la verdad tuve una mala experiencia porque me dieron una habitación alejada y al entrar olía terrible a humedad, (Opinión 14 Hotel C).

(9)

Estamos hospedados en el hotel, vinimos a pasar nuestra luna de miel, desde que llegamos tuvimos problemas. (Opinión 2 Hotel B, viaje en pareja).

Esta formulación alterna asimismo con la presentación orientada al tópico, según el esquema tópico + opinión, que no emplea la primera persona sino que focaliza el texto en la valoración de la experiencia, sin marcas individualizadoras ni apelativas, como puede verse en (10).

(10)

Hotel bastante agradable, pequeño pero con lo mínimo para divertirte, la atención en bares si deja un poco que desear, la calidad de los tragos también es bastante baja. Los restaurantes también cumplen con los mínimo. La ubicación, es lejos del downtown, pero está al costado del puerto a Isla Mujeres (Opinión 9 Hotel A).

En las respuestas de los hoteles, el número apariciones de pronombres de primera persona singular y plural se invierten, con un uso ocasional del "yo" (21 apariciones) en favor del "nosotros" (589 casos), una forma menos referencial que sirve como indicador de cortesía a la vez que expresa el stance corporativo y profesional. Las respuestas de los hoteles se presentan como representantes de empresa y aparecen menos individualizados, ya que lo que dicen no lo hacen a título individual sino como resultado de un trabajo en equipo, tal y como puede verse en (10) y (11). En todo caso, el hotel alterna estratégicamente el uso de las formas singular y plural: nosotros para mostrar la imagen corporativa (en nombre de todos los que trabajamos aquí), subrayando el carácter colectivo de la empresa, mientras que el cambio al singular aporta la fuerza ilocutiva de la individualización. En este caso, la persona encargada de atender al cliente ofrece una disculpa al cliente y muestra su compromiso personal por reparar el daño causado: "le quiero ofrecer la más cordial disculpa" o "le puedo asegurar", subrayando el carácter compromisivo del enunciado.

(11)

Lamentamos que haya sentido que le quitara tiempo, pero le puedo asegurar que el simple hecho de conocer esta información es una inversión para tomar decisiones de alojamiento (Opinión 2 Hotel A)

En nombre todos los que trabajamos aquí quiero ofrecerle la más cordial disculpa por los inconvenientes que tuvo durante su estancia (Opinión 3 Hotel A)

El uso del pronombre de segunda persona, por otro lado, ofrece indicaciones acerca de las audiencias a las que se dirige la reseña. En este sentido, las opiniones de los huéspedes no muestran de forma explícita a quién se dirigen cuando deciden 
publicar su opinión, por lo que ha de entenderse que la reseña no posee una audiencia específica y que por tanto se dirige genéricamente a los usuarios de la plataforma (véase los ejemplos 9 y 10). Por otro lado, se han encontrado casos en los que el autor de la reseña sí se dirige a los lectores, entendiéndose que estos son los viajeros que potencialmente podrían visitar ese hotel y a los que advierte de los inconvenientes (13).

Además, aparece (en 70 casos) el uso del pronombre de segunda persona singular, que muestra una lectura ambigua, ya que puede tratarse de una forma de dirigirse a la audiencia, o bien de una construcción impersonal, que se emplea con frecuencia en la lengua hablada (Enríquez 1984, Hidalgo Navarro 1996-97, Gómez Torrego 1994, De Mello 2000, Guirado, 2011). Los ejemplos encontrados además abundan en reseñas de usuarios que proceden de Argentina, lo que puede indicar un rasgo de esta variedad americana (14). Puesto que el autor de la reseña relata algo del pasado (lo que ha vivido en su estancia en el hotel), puede interpretarse que la construcción impersonal empleada, junto con el tiempo presente, sirve para dotar el relato de dinamismo y actualización o acercamiento.

(13)

Aunque después, cada vez que nos veía nos preguntó con ironía si habíamos visto más cucarachas! Lo que lamentamos es que en ningún momento se nos compensara por las molestias ocasionadas. Espero que os sirva nuestra lamentable experiencia en dicho hotel (Opinión 5 Hotel C)

(14)

Las camas balinesas de la piscina para la opción level estaban ocupadas por quien le apeteciese estar alli y tenias_que reclamar tu mismo, ademas estaban sucias. (Opinión 16 Hotel A)

Por otro lado, los hoteles se dirigen directamente a los autores; no emplean las formas "tú" sino "usted", marcando la distancia social y estableciendo una relación de empresa hacia el cliente, por lo que emplea sistemáticamente formas nominales y pronominales de deferencia y formalidad, como "usted", "le", Sr." "Sra". Mediante estos usos, la empresa hotelera se sitúa en un registro formal y corporativo que se distingue claramente de la esfera del cliente. En (14) puede observarse el contraste entre la reseña y la respuesta: mientras que la primera se construye desde la primera persona y no se dirige a la audiencia de forma específica, el hotel se dirige al cliente con cortesía formal y distanciada, con la finalidad de proyectar una imagen profesional y comercial, que sea leída por su cliente pero también por todos los usuarios de la plataforma, y por tanto potenciales clientes también.

\section{(15) (Opinión 8 Hotel A)}

Es el mejor hotel q he visitado en Cuba, la habitación con excelentes condiciones, las visitas excepcionales, la piscina bella, la zona de playa Caribe 5 estrella, el trato del personal excelente. XXX (nombre del autor de la reseña)

Estimada XXX:

En nombre de todo el equipo de Meliá Marina Varadero nos gustaría agradecerle sus valoraciones tras su reciente visita a nuestro hotel.

Deseamos recibirle de nuevo en un futuro no muy lejano. Me encantaría conocerle personalmente en su próxima visita al hotel, por lo que ruego me dejen saber las fechas de su próxima estancia

Saludos Cordiales

XXX, Community Manager 
En tercer lugar, se ha examinado la aparición de indicadores de cortesía. Para ello, se han computado aquellas expresiones lingüísticas que corresponden con actos de habla formulaicos, como agradecimientos y disculpas (Bunz \& Campbell 2002), en los que se muestra un tipo de cortesía normativa que se expresa con formas convencionalizadas.

Tabla 4. Indicadores de cortesía en reseñas y respuestas de hoteles. Número de apariciones y [ratio de apariciones por reseña] respuestas de hoteles

\begin{tabular}{|l|c|c|c|c|}
\hline Formas & \multicolumn{2}{|c|}{ Reseñas } & \multicolumn{2}{c|}{ Respuestas hoteles } \\
\hline & $\mathrm{N}^{\mathrm{a}}$ & {[]} & $\mathrm{N}^{\mathrm{a}}$ & {[]} \\
\hline Agradecimientos & 30 & {$[0,25]$} & 129 & {$[1,26]$} \\
\hline Disculpas & - & - & 53 & {$[0,52]$} \\
\hline TOTAL & 30 & {$[0,25]$} & 182 & {$[1,78]$} \\
\hline
\end{tabular}

Fuente: Elaboración propia

En nuestro corpus, tal y como puede observarse en la Tabla 4, se advierte una variación importante entre las reseñas y las respuestas. Los agradecimientos aparecen tanto en reseñas como en respuestas, si bien la proporción es más elevada en las respuestas que en las reseñas. La presencia reiterada de este acto de habla revela su importancia como estrategia de cortesía en estas interacciones, ya que remite a la expresión de satisfacción del cliente por los servicios recibidos, así como a la consiguiente satisfacción del hotel por haber logrado complacer al huésped (véase el ejemplo 14). Se trata, por tanto, de una forma que en la interacción marca la reacción y el reconocimiento recíproco tras una experiencia positiva ocurrida fuera de la red, y que se muestra públicamente en ella, indicando que lo que sucede fuera, offline, se refleja dentro, online. Por otro lado, las respuestas de los hoteles contienen disculpas, que aparecen en las respuestas a evaluaciones negativas o quejas de los clientes, y que se presentan como fórmulas de cortesía en las que la empresa se dirige formalmente a los clientes y plantea como acto reparador por el posible daño causado al cliente. En el ejemplo (14), el hotel emplea el agradecimiento como fórmula de respuesta, tras el saludo: mediante este acto de habla, reconoce la importancia de los comentarios depositados por los clientes, especialmente si son positivos (nos gustaría agradecerle sus valoraciones tras su reciente visita a nuestro hotel) por las repercusiones que tiene para la difusión de sus servicios, y por tanto este acto de habla encabeza las respuestas de forma generalizada. El carácter elaborado y formulaico de estos actos de habla, que siguen una estructura compleja característica, remite a la intención de las empresas de ofrecer formas de reparación a los actos de los usuarios, con frecuencia quejas (Márquez Reiter \& Hidalgo Downing 2020, Hidalgo Downing, en preparación).

Por último, se han estudiado las marcas ortográficas y tipográficas de las reseñas y las respuestas de los hoteles. La relajación de este tipo de marcas constituye un rasgo característico de la CMT, según numerosos estudios (Yus, 2011; Herring et.al., 2013; Cantamutto, 2017; Mancera Rueda, 2016). En la CMT, los usuarios emplean distintos recursos que se refieren al modo o canal (Halliday, 1985), ya que suponen mecanismos de adaptabilidad al soporte tecnológico. Además, en consonancia con la evolución de los medios tecnológicos, pueden producirse diferencias importantes entre la comunicación mediada por ordenador, y el uso de dispositivos móviles (Gianmateo, Gubitosi y Parini, 2017). Estos últimos comportan una escritura más rápida e inmediata, con menor planificación. Los dispositivos móviles conllevan predictores de palabras que producen en muchos casos errores tipográficos o palabras erróneas, y pueden carecer además de signos 
ortográficos. La escritura digital por ordenador, en cambio, puede preservar de forma más estable los rasgos de escritura tradicional, normativa. Sin duda estas diferencias se muestran de un modo evidente en las reseñas y las respuestas de los hoteles; en los dos subcorpus se ha computado un conjunto de rasgos que aparecen ya de forma habitual en interacciones digitales, si bien, como veremos, no es un fenómeno generalizado. Estos rasgos son: abreviaturas, errores ortográficos, errores tipográficos, puntuación expresiva (uso de varios puntos de exclamación o de interrogación o puntos suspensivos); el uso de mayúsculas para indicar énfasis, pero también emociones, principalmente negativas, como enfado o indignación; emoticonos o emojis, y oralizaciones, es decir formas escritas que imitan o evocan elementos de la oralidad, como onomatopeyas o interjecciones.

Tabla 5. Marcas ortográficas y tipográficas en reseñas y respuestas de hoteles. Número de apariciones y [ratio de apariciones por reseña] respuestas de hotel

\begin{tabular}{|l|c|c|c|c|}
\hline Formas & \multicolumn{2}{|c|}{ Reseñas } & \multicolumn{2}{c|}{ Respuestas hoteles } \\
\hline & $\mathrm{N}^{\mathrm{a}}$ & {[]} & $\mathrm{N}^{\mathrm{a}}$ & {[]} \\
\hline Abreviaturas & 95 & {$[0,79]$} & - & - \\
\hline Errores ortográficos & 749 & {$[6,24]$} & 24 & {$[0,23]$} \\
\hline Errores tipográficos & 105 & {$[0,88]$} & 20 & {$[0,19]$} \\
\hline Puntuación expresiva & 92 & {$[0,77]$} & - & - \\
\hline Mayúsculas & 242 & {$[2,02]$} & - & - \\
\hline Emoticonos /emojis & 7 & {$[0,06]$} & - & - \\
\hline Oralizaciones & 6 & {$[0,05]$} & - & - \\
\hline & 1296 & {$[10,80]$} & 44 & {$[0,42]$} \\
\hline
\end{tabular}

Fuente: Elaboración propia

La Tabla 5 muestra los resultados del cómputo y avalan la hipótesis general, según la cual reseñas y respuestas muestran estilos radicalmente distintos. En este caso, los autores de reseñas emplean numerosos recursos que han sido identificados por estudios anteriores como característicos de la CMO: emplean abreviaturas, puntuación expresiva, mayúsculas, y cometen numerosos errores ortográficos y tipográficos. La frecuencia de estos recursos sugiere que los autores de reseñas no cuidan particularmente la redacción y edición final de la reseña, que publican de forma inmediata y envían desde sus dispositivos móviles (tal y como se muestra en el encabezamiento de la reseña, que especifica "opinión depositada desde dispositivo móvil”). En particular, los rasgos más frecuentes son los errores ortográficos y tipográficos, lo que puede considerarse un rasgo asociado al modo o canal, un rasgo por tanto de adaptabilidad, en el sentido de que el soporte (dispositivo móvil) condiciona ese rasgo estilístico. Otro factor importante, asociado al primero, es la ausencia de planificación. Estos rasgos reflejan que la escritura digital de la reseña es inmediata y espontánea, y carece de planificación. Otros rasgos interesantes son los emoticonos/emojis y oralizaciones, que también aparecen pero en frecuencias mucho menores. Es un dato muy destacable porque aleja el estilo de las reseñas de otras interacciones digitales, como las que se desarrollan en redes sociales (Facebook, Instagram, twitter) o WhatsApp, en los que proliferan y abundan cada vez más. 
Sin duda, este rasgo remite al carácter no interactivo de la reseña. Los emoticonos y emojis y oralizaciones que se emplean sirven como intensificadores, en general de valoraciones positivas, pero no realizan funciones interactivas, como sí ocurre en redes sociales. Otro factor sin duda importante es la distancia social, en cuanto en la plataforma TripAdvisor los usuarios no se conocen entre sí, y por tanto no muestran la interactividad afectiva de las redes sociales.

La Tabla 5 muestra que el cómputo en las respuestas de los hoteles resulta opuesta.

Tan solo ofrecen casos de errores ortográficos y tipográficos, que pueden explicarse en virtud de los conocimientos y competencias comunicativas de las personas designadas por los hoteles para gestionar la comunicación digital, como el dominio de la lectura y escritura, su grado de instrucción y de competencia escrita, o la falta de revisión del texto final (corrector gramatical). Se entiende que la ausencia de abreviaturas, así como el uso de la puntuación convencional, remite al hecho de que las respuestas de los hoteles remiten a un modelo de cara escrita, y no constituyen realmente ejemplos de escritura digital, ya que no muestra rasgos de adaptabilidad al soporte tecnológico (16).

(16) Opinión 7 Hotel C

Pésimo!!!!!!

El peor hotel y además es carísimo las habitaciones de cuarta!!!!! Pierden agua por todos lados,los servicios horribles la piscina es para 5 personas como mucho y los bares un desastre no es ni 3 estrellas este hotel perdimos tiempo y Plata cambiando de hotel el show una vergüenza no lo recomiendo para nada!

Estimado cliente,

¡Le agradecemos que nos haga llegar sus impresiones.

Sentimos mucho que en general su experiencia con nosotros no fuera lo que usted esperaba y que haya dejado nuestro hotel con una impresión totalmente opuesta a la que procuramos transmitir siempre.

Nos gustaría poder recibirle de nuevo en uno de nuestros hoteles.

Un cordial saludo,

Hotel C

Online Reputation Manager

Así en (16) puede apreciarse la divergencia entre la reseña y la respuesta. Mientras que la primera carece de signos de puntuación, lo que refleja ausencia de planificación y de revisión, así como la escritura desde un dispositivo móvil, también emplea los puntos exclamativos múltiples para aportar expresividad, lo que constituye un rasgo extendido en la escritura digital. El hotel, en cambio, no emplea rasgos propios de la escritura mediante dispositivo y revela una redacción cuidada. El empleo de un estilo formal y elaborado, en el que además se cuida la edición final del texto, entra en consonancia con la imagen de profesionalidad que desea transmitir el hotel.

\section{Variación de estilo y norma lingüística}

El corpus de reseñas y respuestas de hoteles ha sido examinado desde distintos parámetros que han permitido observar la variación de estilo que presentan estas interacciones, y en particular la distinción entre las reseñas y las respuestas. Los resultados han mostrado que existe variación importante en todos los parámetros estudiados, y que por tanto no se 
puede ofrecer una visión general de la lengua que aparece en este tipo de comunicación digital. Por el contrario, en el análisis se ha podido comprobar que la variación de estilo responde a un complejo conjunto de factores que intervienen en la situación comunicativa, como el diseño del destinatario o público receptor, la acomodación al otro, el prestigio, tal y como mencionaba Garrido Medina (1997: 117) en su estudio sobre el estilo en la lengua. Además, cabe destacar la noción de adaptabilidad de Verschueren (1999) a las posibilidades y limitaciones (lo que denominan "affordances") del medio. Así pues, el estilo de reseñas y respuestas se muestra divergente o incluso contario en los puntos siguientes: (i) direccionalidad, (ii) formalidad y (iii) planificación, que reflejan, tal y como decía, Herring, la presencia de distintos actores sociales. Asimismo destacable es la variación en cuanto a la presencia de usos normativos, tanto como una distinta presencia u observación de la norma.

La direccionalidad de reseñas y respuestas se ha mostrado divergente, ya que la reseña se construye en torno a dos esquemas posibles, similares pero variables, que son tópico + opinión, o bien yo + opinión, sin la mención de un lector específico al que se dirige, y que se entiende es el usuario de la plataforma, u otros viajeros. Esta característica muestra que la finalidad principal de la reseña es valorar la estancia en el hotel, y recomendar o no a otros viajeros; la relación que establece con esos potenciales lectores es de igualdad o solidaridad, por lo que se impone el tratamiento informal. El hotel, en cambio, construye su respuesta desde un nosotros que representa el equipo del hotel y marca el stance corporativo; se dirige al cliente con un tratamiento formal y elaborado, que proyecte una imagen de profesionalidad y dominio de los usos normativos, formales de la lengua. Estos rasgos se emparentan tanto con el locus (el campo) como con el tenor funcional e interpersonal.

La formalidad constituye un rasgo que caracteriza las respuestas de los hoteles recogidos en el corpus. En los estudios sobre comunicación mediada por tecnología, se insiste casi de forma sistemática en la informalidad como rasgo distintivo de este tipo de comunicación, tanto en las referencias sobre el inglés como del español. En nuestro corpus, en cambio, las empresas hoteleras escogidas se dirigen al cliente mediante un registro formal, que contrasta con el empleado por el usuario. Se puede pensar que estas empresas pretenden, con ello, ofrecer una imagen profesional y corporativa.

Es decir, el hotel busca los atributos que el lector pueda identificar de forma inequívoca con un lenguaje formal, como el empleo de formas de cortesía de deferencia (usted/le/Sr./Sra.), fórmulas de cortesía y elaboración textual. La respuesta del hotel se enmarca en un modelo previo y externo al medio digital, el de la carta comercial, y se presenta como una forma marcada con respecto a la reseña y al medio, y responde a la finalidad de proyectar de nuevo una imagen profesional y corporativa (Suau Jiménez 2019). La reseña, en cambio, muestra rasgos de informalidad, pero en distinto grado, lo que sugiere un planteamiento más espontáneo o sin un modelo claro que seguir, que dependerá del usuario (su competencia de escritura digital y nivel de uso que desee aportar). En este sentido, este trabajo aporta resultados nuevos, que contrastan con estudios anteriores. Así, el trabajo de Cantamutto (2107) sobre mensajes SMS que empleaban empleados y superiores en un entorno laboral, mostraba que estos se servían de un estilo fundamentalmente informal y coloquial. También los estudios realizados sobre respuestas de empresas a los clientes en plataformas digitales chinas (Feng \& Ren 2019) encontraron que las empresas se dirigían al cliente con un lenguaje informal y cercano, con el que la empresa intentaba establecer una relación de proximidad y mitigar posibles errores en el servido. Al contrario, nuestros datos han mostrado que las empresas hoteleras escogidas se dirigen al cliente mediante un registro formal. 
Por último, la planificación constituye un eje sobre el que puede observarse el distinto planteamiento de los autores de las reseñas y los hoteles. Los autores escriben sus reseñas con frecuencia desde dispositivos móviles y muestran escasa planificación en la elaboración del texto, con la aparición además de numerosos rasgos ortotipográficos que remiten a la escritura digital mediante dispositivos, como los errores tipográficos (o bien por descuido del autor, o bien por el corrector del dispositivo) y ortográficos. La puntuación expresiva (uso múltiple de puntos de interrogación o exclamación), así como las abreviaturas, remiten a usos que se van consolidando en la escritura digital, al menos la que se desarrolla mediante dispositivos electrónicos. Otros elementos típicos, como los emoticonos, emojis, no aparecen aquí, lo que refleja de nuevo, la distancia social entre los usuarios, que no se conocen entre sí. En el otro extremo, las respuestas de los hoteles reflejan un estilo elaborado con un modelo previo, que remite a formas prestigiadas de la lengua. La repetición de las mismas fórmulas remite además a modelos textuales que los hoteles emplean a modo de modelos de carta o plantillas sobre las que construyen un texto claramente identificable.

En cuanto a la relación con la norma lingüística, en las producciones de los usuarios podemos analizar los dos conceptos de norma lingüística. En el sentido prescriptivo, las respuestas de los hoteles sin duda muestran claramente un registro formal que se adecua a la norma, o que lo intenta, ya que la presencia de inconsistencias ortográficas y tipográficas indica niveles distintos de conocimientos de la lengua escrita y la norma culta. Pese a esta variación casi involuntaria, se percibe que el modelo lingüístico es el normativo. Por otro lado, los autores de las reseñas no muestran un modelo lingüístico ajeno al empleado fuera de la red, sino un estilo que entra plenamente en lo que podríamos caracterizar como escritura digital. En el caso de estas reseñas, la escritura digital comporta la escritura desde dispositivos móviles, la escasa o inexistente planificación del texto, así como la ausencia de mecanismos de revisión o edición del texto. En esta forma espontánea y plenamente adaptada de escritura al medio, el usuario no se remite a un modelo fuera de la red sino a los usos que ya han aparecido y se han extendido en el español en la red: el uso de las abreviaturas, por ejemplo, la relajación y aceptación en los errores tipográficos y el empleo de la puntuación expresiva: el uso de varios puntos de exclamación o interrogación. De acuerdo con la ortografía actual de la RAE, solo se consideraría correcto el uso doble del punto de exclamación, para dotarlo de mayo expresividad. El uso abundante de las formas exclamativas e interrogativas refleja que los usuarios no tienen en mente la norma lingüística prescriptiva de la RAE, que quizá desconocen (dominio de la lectura y escritura o literacy), pero sí tienen en cuenta una serie de usos que se han extendido y consolidado en la red, y que por tanto remiten a esa norma en el sentido descriptivo, de lo que se ha convertido en "normal" o "usual" en las interacciones digitales. En este sentido, esos usos pueden con el tiempo convertirse en los rasgos aceptados de una escritura digital, que no tiene un modelo externo a la red, sino que supone la consolidación de esta forma de escritura.

\section{Conclusiones}

El análisis de las reseñas y respuestas de hoteles en TripAdvisor revela un grado importante de variación de estilo, en todos los parámetros estudiados, saludos y despedidas, formas de tratamiento y rasgos ortotipográficos, lo que sugiere que unos y otros participan en la plataforma como "actores sociales" con realidades sociales distintas (Herring et. al. 2013) y que emplean la plataforma con finalidades comunicativas y diseños de audiencias distintos: los prosumidores con la 
finalidad de valorar sus experiencias en los hoteles, y las empresas en cambio con la intención de mantener o mejorar su reputación empresarial a través de una reacción a sus comentarios. Además, la diferencia en los rasgos estilísticos muestra grados muy distintos de competencia escrita y de percepción de la norma lingüística, según los modelos que los actores sociales tienen presentes. No obstante, se trata de un primer estudio sobre un producto concreto (reseñas de hoteles de calidad media y alta), que habría que contrastar con otros productos u otros tipos de alojamientos. Por tanto, los resultados del análisis no eran previsibles; al contrario, los estudios previos mostraban que las interacciones digitales, también en el ámbito de empresa-cliente, prefieren estilos informales y cercanos. En este sentido, este trabajo aporta resultados nuevos, sobre los que se habrá que seguir indagando, para averiguar si se trata de una característica del producto escogido, de la lengua-cultura o de otros factores.

La noción de norma en la comunicación mediada por tecnología ha de aplicarse al análisis de los estilos y registros que se asocian a prácticas discursivas concretas; en ellas, podrá verse en qué medida el principio de adaptabilidad (Virtannen 2017) crea hábitos lingüísticos que se consolidan en la comunicación y que otros usuarios reconocen hasta el punto de constituirse en convenciones discursivas. No obstante, esos nuevos hábitos no dejan de transmitir /ser índices de información sociolingüística y sociopragmática, por lo que los usuarios proyectan imágenes de sí a través de esos estilos. Por todo ello, el estilo comunicativo que emplean los usuarios en la red es particularmente significativo y relevante en la configuración de lo que puede ser "normal" o "habitual" en la escritura digital, y quizá llegar algún día a formar parte de una norma codificada para la comunicación mediada por tecnología. En palabras de Méndez García de Paredes, "la costumbre se hace precepto cuando se codifica, y una vez hecha norma, se adopta como elemento de juicio y establece el modelo de lo que debe ser, es decir funciona como norma ejemplar" (Méndez García de Paredes 1999: 111).

\section{Referencias bibliográficas}

Albaladejo, T. (2010). La poliacroasis y su manifestación en la retórica política. A propósito del discurso inaugural de Bararck Obama (pp. 927-939). En J. L. Cifuentes, A. Gómez, A. Lillo, J. Mateo, E. Yus (eds.), Los caminos de la lengua. Estudios en homenaje a Enrique Alcaraz Varó. Alicante: Universidad de Alicante.

Briggs, Senga, Sutherland, Jean, Drummond, Siobhan, 2007. Are hotels serving quality? An exploratory study of service quality in the Scottish hotel sector. Tourism Management 28, 1006-1019.

Briz, A. (1998). El español coloquial en la conversación. Esbozo de pragmagramática. Barcelona: Ariel.

Browning, V. So, K. \& Sparks, B. A. (2013). The Influence of Online Reviews on Consumers' Attributions of Service Quality and Control for Service Standards in Hotels, Journal of Travel \& Tourism Marketing, 30 (1-2) 23-40.

Bunz, U. y Campbell, S.W. (2002). Accomodating politeness indicators in personal mail messages. Association Internet Researchers, Maastrich, Países Bajos. https://pdfs.semanticscholar.org/c14a/250fc1100b13f33c97b35b5bf6eab39d6f7d.pdf Cantamutto, L. (2017). Economía, claridad y expresividad lingüísticas: el estilo comunicativo digital del teléfono móvil en el español bonaerense. En Gianmateo, M., Gubitosi, P. y Parini, A. (Eds.), El español en la red. Madrid: Iberoamericana Vervuert, 93-123. 
Chen, Y. \& Xie, J. (2008). Online consumer review: Word-of-Mouth as a new element of Marketing Communication Mix. Management Science, 55 (3), iv-626.

De Benito Moreno, C., Estrada Arraz, A. (2018). Aproximación metodológica al estudio de la variación lingüística en las interacciones digitales, Revista Estudios del Discurso Digital (REDD), 1, 74-122. DOI: https://doi.org/10.24197/ redd.1.2018.74-122

DeMello, George. (2000). “Tú” impersonal en el habla culta. Nueva Revista de Filología Hispánica 48. 2, 359-372.

Enríquez, Emilia. (1984). El pronombre personal sujeto en la lengua española hablada en Madrid. Madrid: CSIC.

Feng, W. \& Ren, W. (2019). "This is the destiny, Darling”: Relational acts in Chinese management responses to online consumer reviews. Discourse, Context \& Media 28, 52-59.

Garrido Medina, J. (1997). Estilo y texto en la lengua. Madrid, Gredos.

Gee, J. P. (1999). Discourse Analysis: Theory and Method. Londres, Routledge.

Gobierno de España (2019). Boletín del Ministerio. Ministerio de Industria, Comercio y Turismo. https://www.mincotur. gob.es

Gómez Torrego, Leonardo. (1994). La impersonalidad gramatical: descripción y norma. Madrid: ArcoLibros

Goffman, Erving (1981). Forms of Talk. Philadelphia: University of Pennsylvania Press.

Guirado, Krístel. 2011. Uso impersonal de tú y uno en el habla de Caracas y otras ciudades. Círculo de Lingüística Aplicada a la Comunicación 47, 3-27.

Halliday, M.A.K. (1985). El lenguaje como semiótica social. México: Fondo de Cultura Económica.

Hernández López, M. O. (2019). What makes a positive experience? Offline/online communication and rapport management in Airbnb positive reviews. Pragmatics and Society 10:2, 177-204.

Hernández Toribio, M. I; Mariottini, L. (2015). Actos de habla y atenuación 2.0: TripAdvisor. Círculo de Lingüística Aplicada a la Comunicación, 73, 15-32.

Herring, S., Stein, D., Virtanen, T. (2013), Introduction to the pragmatics of computer-mediated communication. En Herring, S., Stein, D., Virtanen, T. (Eds.) Handbook of Computer-Mediated Communication. Berlín: Mouton, 3-31.

Hidalgo Navarro, Antonio. (1996-97). Sobre los mecanismos de impersonalización en la conversación coloquial: el tú impersonal. Estudios de Lingüística 11, 163-176.

Law, R. (2006). Internet and tourism. Part XXI. TripAdvisor. Journal of Travel \& Tourism Marketing, 20 (1), 75-77.

Mancera Rueda, A. (2018), La atenuación lingüística en las reseñas digitales de hoteles y restaurantes en español, Círculo de Lingüística Aplicada a la Comunicación, 73, 53-76.

Mancera Rueda, A. (2016). Usos lingüísticos alejados de la norma como señas de identidad en las redes sociales. Bulletin of Spanish Studies DOI: 10.1080/14753820.2016.1181435.

Mariottini, L. \& Hernández Toribio, M. I (2017). La narración de experiencias en TripAdvisor. RILCE: Revista de Filología Hispánica. Volumen 33 (1), 302-330. 
Márquez Reiter, R. \& Hidalgo Downing, R. (2020). Intercultural communication in a globalized world. En D. Koike \& C. Félix-Brasdefer (eds.), Handbook of Spanish Pragmatics. Londres, Routledge, 305-320.

Martín Zorraquino, M. A. (1988). Norma, gramaticalidad, aceptabilidad... reflexiones sobre la delimitación del objeto lingüístico a propósito de conceptos acuñados por Eugenio Coseriu. Energeia und Ergon. Sprachliche VariationSprachgeschichte-Sprachtypologie. Studia in honorem Eugenio Coseriu, 2, 431-440.

Méndez García de Paredes, E. (1999). La norma idiomática del español. Visión histórica. Philologica Hispalensis 13, 109-132.

Miguéns, J., Baggio, R., Costa, C., (2008). Social media and tourism destinations: TripAdvisor case study. In: IASK ATR2008 (Advances in Tourism Research 2008), Aveiro, Portugal, May 26-28.

Ngwenyama, O.K. \&Lee, A.S. (1997). Communication richness in electronic mail: Critical social theory and the contextuality of meaning. MIS Quarterly21, 145-167.

Pérez Sabater, Carmen, Turney Ed., Montero Fleta, Begoña. (2008). Orality and literacy, formality and informality in email communication. Ibérica 15, 71-88.

Portolés, José (2004). Pragmática para hispanistas. Barcelona: Síntesis.

Schwab, Pierre-Nicolas and Rothenberger, Sandra. 2015. Online Complaint Handling: The Effects of Politeness and Grammaticality upon Perceived Professionalism and Loyalty. Centre Emile Bernhein (CEB) Working Paper N 15/015 April 2015

Sparks, Beverly A., and Victoria Browning. 2010. “Complaining in cyberspace: the motives and forms of hotel guests' complaints online.” Journal of Hospitality Marketing \& Management 19(7): 797-818. https://doi.org/10.1080/19368623.20 10.508010

Suau-Jiménez, F. (2019). How hotel websites may discursively adjust to customer preferences using online criticism. Ibérica 38, 203-226.

Vásquez, C. (2011). Complaints online: The case of TripAdvisor. Journal of Pragmatics 43(6),1707-1717.

Vásquez, C. (2014): The Discourse of Online Consumer Reviews. Londres: Bloomsbury.

Vela Delfa, C. (2016). Una aproximación del correo electrónico desde una perspectiva diacrónica: evolución y asentamiento de un género discursivo. Cadernos de Linguagem e Sociedade 17 (2), 55-78.

Verschueren, J. (1999). Understanding Pragmatics. Londres/Nueva York: Oxford University Press.

Virtanen, T. (2017). Adapatibility in online consumer reviews: Exploring genre dynamics and interactional choices. Journal of Pragmatics, 116, 77-90.

Yus, F. (2011). Ciberpragmatics. Internet-mediated communication in context. Amsterdam, John Benjamins.

Zhang, y. \& Vasquez, C. (2014). Hotel responses to online reviews: managing consumer dissatisfaction. Discourse, Context and Media 6, 54-64. 